\title{
Noninvasive Uric acid Monitoring Device using Near-Infrared Spectroscopy
}

\section{Jae Kim*}

University of Central Florida College of Medicine, 6850 Lake Nona Blvd, Orlando, FL 32827, USA

\begin{abstract}
The prevalence of gout in the United States has risen over the last twenty years and gout now affects 8.3 million Americans. Currently, gout is diagnosed either by extracting a fluid sample from the joint to look for formation of uric acid crystals or by drawing blood to measure the level of uric acid. Both of these methods are invasive, cumbersome, and time-consuming. The purpose of this study is to prevent gout attack with a novel approach called "noninvasive infrared uric acid monitoring." This approach utilizes a cost-effective and portable near-infrared (NIR) spectroscopybased device that can offer quick, noninvasive, and preventative way to monitor the patient's uric acid level.

By using uric acid infrared spectroscopy characteristics, infrared wavelengths between 1400 to $1700 \mathrm{~nm}$ are emitted on uric acid samples and detector senses non-absorbed Infrared light. The detected NIR signal gets amplified and filtered to maintain a high signal to noise ratio (SNR) over the wavelengths of interest. A linear regression algorithm is used to distinguish and predict the uric acid concentration among other biomarkers in the sample. Finally, a digital signal processing was used to process the data.

Based on the Beer-Lambert's law, a linear relationship between the light absorbance and uric acid concentration is expected. Minimum detectable change in uric acid level was analyzed and a mathematical model was compared to the measured data.
\end{abstract}

Another potential benefit of this approach is its versatility. By modifying infrared wavelength and the calibration system in the DSP chip, other biomarkers can be measured.

Keywords: Uric acid; Gout; Near-Infrared Spectroscopy (NIR); Noninvasiveness; Biosensor

\section{Introduction}

Hyperuricemia is an alarming condition, as it can lead to many detrimental complications. Such complications include gout, kidney stones, cardiovascular disease (CVD), and Type 2 diabetes [1]. Among these, gout is the most prevalent complication, and it occurs when uric acid builds up in the blood and causes inflammation in joints [2]. Today, about 8.3 million Americans suffer from gout and there is no viable cure. Patients can only manage their uric acid levels to prevent gout attacks. In addition to gout, recent publications discuss a possible relationship between hyperuricemia and cardiovascular disease, although it is uncertain whether hyperuricemia is an independent or dependent risk factor for CVD [3]. Since there is a direct correlation between blood uric acid level and risk for complications, [4] it would be ideal for patients to have an easy method to measure their uric acid level frequently and comfortably to prevent recurrence of complications due to hyperuricemia. Therefore, it is important to evaluate current diagnostic methods and understand the advantages and disadvantages associated with each method.

Today in the United States, about $21.2 \%$ of men and $21.6 \%$ of women are considered hyperuricemic [5]. Hyperuricemia can be categorized as either primary or secondary. Primary is involved with hereditary or inborn error while the secondary is an acquired disorder. The mechanism of hyperuricemia is the same in both categories; it is due to either increased turnover of nucleic acid purines or to a renal impairment in the excretion of uric acid [6].

Uric acid is the final product of metabolic breakdown of purine nucleotides and it is a heterocyclic compound of carbon, nitrogen, oxygen and hydrogen with the formula $\mathrm{C}_{5} \mathrm{H}_{4} \mathrm{~N}_{4} \mathrm{O}_{3}$. Normally, the rate of uric acid being excreted through the kidney is balanced by the rate that uric acid is being produced in the body. However, when the rate of uric acid production exceeds that of excretion, the homeostasis will be disturbed and the condition is called 'hyperuricemic' where higher than normal net uric acid will accumulate in the body. According to the National Institutes of Health, normal blood uric acid level ranges from 3.5 to $7.2 \mathrm{mg} / \mathrm{Dl}$ [7].

Currently, to measure uric acid or confirm a gout attack, either blood is drawn from the patient or uric acid crystals are aspirated for further analysis, as shown in Figure 1a [8]. Major drawbacks associated



(a) Aspiration of Uric Acid Crystals



(b) DECT (Urate Deposition in Big Toes)
Figure 1: Current Methods to assess uric acid crystals: (a) Aspiration (b) DECT.

${ }^{*}$ Corresponding author: Jae Kim, University of Central Florida College of Medicine, 6850 Lake Nona Blvd, Orlando, FL 32827, USA, Tel: (407) 266-1000; E-mail: jaekim@knights.ucf.edu

Received October 16, 2015; Accepted November 10, 2015; Published November 20, 2015

Citation: Kim J (2015) Noninvasive Uric acid Monitoring Device using Near-Infrared Spectroscopy. J Biosens Bioelectron 6: 188. doi:10.4172/2155-6210.1000188

Copyright: (c) $2015 \mathrm{Kim}$ J. This is an open-access article distributed under the terms of the Creative Commons Attribution License, which permits unrestricted use, distribution, and reproduction in any medium, provided the original author and source are credited. 
with this method include its invasiveness, necessity for presence of a professional to perform the task, the lengthy time frame of obtaining results, and the high cost incurred. Being invasive means that a needle has to penetrate the skin in order to collect the sample. Invasive procedures are usually very painful, cumbersome, and require welltrained professionals. Once the blood or crystal samples are collected, they are sent to the lab for further analysis. Repetition of this procedure over a period of time can be costly. Therefore, this conventional method is not an ideal preventative tool.

In 2010, as an alternative to the conventional method, a noninvasive CT scan-based dual-energy computed tomography (DECT) was introduced to identify uric acid deposits. DECT is an advanced imaging method that enables visualization of uric acid crystal deposits by analysis of the chemical composition of the scanned materials [9]. Figure $1 \mathrm{~b}$ shows an example of the DECT capturing uric acid deposits around the joints of the big toes. A major advantage of DECT over the conventional method is its non-invasiveness. However, CT is neither a cost-effective nor a practical option for the purposes of continuous monitoring. Therefore, like the conventional method, it has its limitations to serve as a preventative tool.

As a potential solution to aforementioned limitations, the purpose of this project was to design and explore a near-infrared spectroscopybased sensor to measure the uric acid levels in the solution that would potentially mimic the composition of the human blood. There are three goals to this project: first, to build a cost-effective IR-based sensor which would respond differently depending on the concentration of the sample of interest; second, to identify if the prototype device can measure uric acid concentration in a linear fashion; and third, to have the device properly distinguish and measure uric acid levels amongst other constituents, which would be albumin protein for this project. Verification of aforementioned goals can be used to check the feasibility of the prototype as a potential portable, noninvasive and cost-effective device to measure uric acid levels in the blood.

Successful implementation and verification of the prototype carries major significance in that the portable, non-invasive, and cost-effective nature of this project will potentially be a major benefit to patients who are either at risk for, or suffer from, complications associated with hyperuricemia. The sensor will be small in size so it will allow patients to carry it to monitor their uric acid levels as needed. With an ability to monitor uric acid levels, patients can take appropriate adjustments (e.g., through diet) early on to prevent severe gout attacks. A significant cost reduction is expected as well because infrared-based sensors can be built very cost effectively and, like any other types of electronics, it can be used continuously without any additional cost. Table 1 summarizes the comparison between existing methods and the proposed device.

\section{Methods and Procedure}

Infrared refers to the part of the electromagnetic spectrum between

\begin{tabular}{|l|c|c|c|}
\hline & $\begin{array}{c}\text { Conventional } \\
\text { Method (Joint } \\
\text { Aspiration and } \\
\text { Blood Test) }\end{array}$ & $\begin{array}{c}\text { DECT (Duel-Energy } \\
\text { CT SCAN) }\end{array}$ & $\begin{array}{c}\text { Proposed Device } \\
\text { (Infrared) }\end{array}$ \\
\hline Checking & $\begin{array}{c}\text { Crystals and uric acid } \\
\text { level }\end{array}$ & $\begin{array}{c}\text { Uric acid crystal } \\
\text { deposition }\end{array}$ & Uric acid level \\
\hline Methods & Invasive & Noninvasive & Noninvasive \\
\hline Portable & No & No & Yes \\
\hline Cost & Expensive over time & Expensive & Economical \\
\hline Accuracy & Very accurate & $\begin{array}{c}\text { Technology } \\
\text { dependent }\end{array}$ & $\begin{array}{c}\text { Technology } \\
\text { dependent }\end{array}$ \\
\hline
\end{tabular}

Table 1: Comparing Conventional Method, DECT and Proposed Device. the visible and microwave regions, as shown in Figure 2. Photon energies associated with infrared region are not large enough to excite electrons, but can induce vibrational excitation on bonds which make up the molecule.

For each molecule, its unique molecular structure allows different response to the IR spectrum. Such difference constitutes different resonant wavelengths for different molecules. For uric acid, the resonant wavelength is near $1700 \mathrm{~nm}$, while cholesterol's resonant wavelength is near $2900 \mathrm{~nm}$ as shown in Figure 3. Knowing resonant wavelength is crucial since molecules respond most sensitively at their resonant wavelengths. The sensor for uric acid was built around wavelength $1600 \mathrm{~nm}$, while the sensor for cholesterol would be built around 3200 $\mathrm{nm}$. Because sensors are built around specific resonant wavelength, one sensor specific for a certain biomarker may not be effective for another.

Figure 4 shows the basic scheme behind an infrared spectroscopy sensor. Infrared light at a resonant wavelength is emitted to the sample and a detector on the other side measures the light passed through the sample. Based on the amount of measured light, the concentration of the sample can be deduced. For this project, the light emitter and detector were built near the resonant wavelength of uric acid.

Many applications have used infrared spectroscopy for biomedical applications, including pulse oxymetry [10] and glucose sensors [11]. They all share the same IR spectroscopy principle, but resonant frequencies used for these sensors are different. For pulse oxymetry, wavelengths $650 \mathrm{~nm}$ and $850 \mathrm{~nm}$ were used while wavelength $2200 \mathrm{~nm}$ was used for glucose sensor. Therefore, for uric acid, the sensor will be built around its resonant wavelength, which is around $1600 \mathrm{~nm}$, as shown in Figure 3.

This project occurred in four phases. Phase 1 was designing the overall architecture and choosing emitter wavelengths for the sensor based on uric acid IR characteristics. Phase 2 was designing the sensor based on the overall proposed architecture from phase 1. Phase 3



Figure 2: Electromagnetic Spectrum.



Figure 3: IR Spectrum of Uric Acid and Cholesterol.



Figure 4: Basic Infrared Spectroscopy Scheme. 
was preparing uric acid solutions and taking IR energy absorption measurements. Uric acid solutions with different concentrations were prepared and the sensor designed from phase 2 was used for measurements.

\section{Phase 1-Choosing overall architecture and wavelength}

Figure 5 shows the overall architecture of the sensor for this project. Infrared light was emitted through the capillary tube with uric acid solution. The detector absorbed the light that passed through the sample and converted it to an electrical voltage. The voltage level will be in millivolts. Therefore, a signal amplifier and filter was used to amplify the signal and filter noise from the environment. Based on the amplified signal, computation block calculated the uric acid level in the solution and displayed the result.

Figure 6 shows the IR spectrum of uric acid. As shown, wavelengths from 1500 to $1700 \mathrm{~nm}$ show the highest wavelength absorption. In other words, a change in light absorbance for changes in uric acid concentration (sensitivity) would be the highest around this region. Therefore, an emitter with wavelength around 1400nm-1700nm was selected.

\section{Phase 2-Circuit design and data collection}

Figure 7 shows the overall circuit implementation of the architecture introduced in Phase 1. Infrared emitter LED1600P was



Figure 5: Overall Architecture of the sensor.



Figure 6: IR Spectrum of Uric Acid



(1) (2) (3)

(5)

(6)

Infrared Emitter (1650nm)

Uric Acid Solution in a test tube

Photodiodes (range: $800 \mathrm{~nm}-1750 \mathrm{~nm}$ )

AC Decoupling Capacitance
Signal Amplifier

6. Analog to Digital Converter and Digital Signal Processing Circuit (PSOC5LP)

a.Phase 3 - Calibration and Measurement

Figure 7: Circuit implementation of the Overall Architecture.



Figure 8: Calibration stage with air and capillary tube.

used (purchased from Thorlab) as it has the highest emitter power around $1650 \mathrm{~nm}$. For the detector, PiN photodiode (from Digikey) was used whose detection range is from $800 \mathrm{~nm}$ to $1750 \mathrm{~nm}$. Once the light is detected, $1 \mathrm{uF}$ capacitor was used as a de-coupling capacitor to filter out the DC component of the electrical signal. Then PSOC5LP was used to amplify the signal and compute the uric acid concentration in solution. Oscilloscope and Multimeter were used to monitor the voltage levels of the circuits during the design process. Uric acid sample and bovine serum albumin were purchased from Sigma-Aldrich.

Before measuring the uric acid solutions, the sensor needs to be calibrated. The calibration stage consists of two steps. First, air (no sample) was used to measure the maximum detectable infrared energy level as shown by Figure 8. During the second part of the calibration, capillary-tube without uric acid and capillary-tube with solvent (water) was used to measure the detectable infrared energy level. This value was compared against data measured from calibration stage part 1. Then, energy absorbed by the capillary-tube was calculated and was subtracted from the total absorption to isolate the energy absorption from the solution.

Different uric acid solutions were prepared and light absorbance was measured. Light absorbance from the test-tube and vacuum is then subtracted to extract the light absorbance by the uric acid sample alone. Data was collected and sensitivity was analyzed. Normal uric acid concentrations in the blood ranges from $3.5 \mathrm{mg} / \mathrm{dL}$ to $7 \mathrm{mg} / \mathrm{dL}$. To mimic the actual blood concentration of the uric acid, samples were prepared from $3 \mathrm{mg} / \mathrm{dL}$ to $7 \mathrm{mg} / \mathrm{dL}$ with an increment of $1 \mathrm{mg} / \mathrm{dL}$. Thus, total of 5 samples were prepared: $3,4,5,6,7 \mathrm{mg} / \mathrm{dL}$.

Solutions containing both uric acid and bovine albumin were prepared and light absorbance was measured. Albumin is chosen as it is the most abundant protein in our serum. Albumin protein in human serum ranges from $3400 \mathrm{mg} / \mathrm{dL}$ to $5400 \mathrm{mg} / \mathrm{dL}$. Such concentration is about 1000 times higher than that of uric acid. Therefore, great sensitivity and selectivity for uric acid would be very crucial. Albumin levels used for the test purpose were divided into four levels: 3400 $\mathrm{mg}, 4400 \mathrm{mg}, 5000 \mathrm{mg}$ and $5500 \mathrm{mg}$. Solutions were homogenously mixed for the linear extraction. Based on the light absorbance data, concentration information of uric acid were extracted by using spectral characteristics of uric acid and albumin protein. This is to verify how uric acid information can be extracted in the presence of other interference molecules in the mixture. Figure 9 shows schematic and prototype device designed to measure the sample.

\section{Results and Analysis}

\section{Light absorption vs concentration for different wavelength}

Wavelength between 1200-1700 $\mathrm{nm}$ were selected. Within this range, four wavelength emitters $(1200 \mathrm{~nm}, 1300 \mathrm{~nm}, 1450 \mathrm{~nm}$ and $1550 \mathrm{~nm}$ ) were used to verify the sensitivity of the device. 


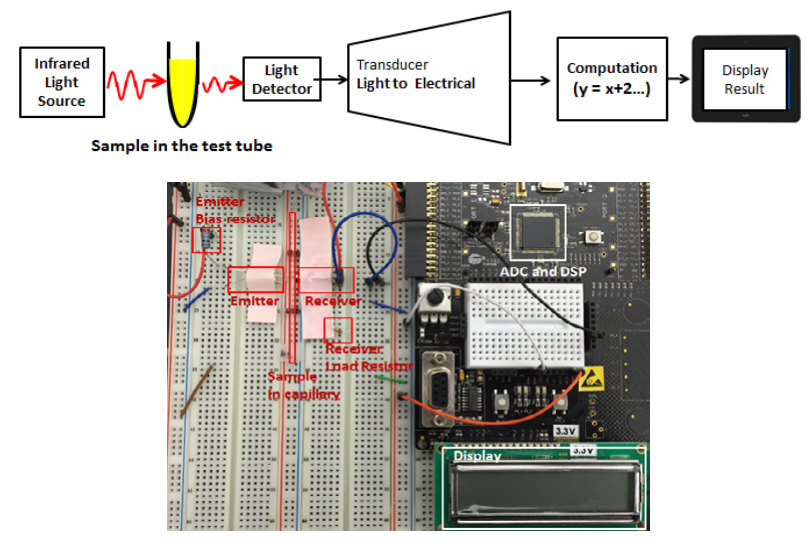

Figure 9: Uric acid sample measurement.



Figure 10: Change in Voltage vs Uric Acid Concentration.

Figure 10 shows the increase in light absorption as the uric acid concentration increase for different wavelengths. As predicted by the Figure 6, wavelengths around 1450-1550 nm are more sensitive compared to $1200 \mathrm{~nm}$ or $1300 \mathrm{~nm}$. Based on Beer-Lambert's law, a linear relationship is expected between light absorption and solute concentration and Figure 10 shows such relationship.

Figure 11 shows the sensitivity of the uric acid over different wavelengths. Average sensitivity (change in light absorption/change in concentration) for each wavelength was calculated based on Figure 10. It can be seen that as wavelengths diverge from $1450 \mathrm{~nm}$, the sensitivity decreases as expected.

Uric acid sensitivity vs protein albumin sensitivity around wavelength at $1450 \mathrm{~nm}$ and $1550 \mathrm{~nm}$

As shown in Figure 11, uric acid is most sensitive at wavelengths $1450 \mathrm{~nm}$ and $1550 \mathrm{~nm}$. Therefore, it is meaningful to explore the sensitivity of the albumin at these wavelengths. Figure 12 shows the uric acid sensitivity and Figure 13 shows the sensitivity of the albumin at wavelengths $1450 \mathrm{~nm}$ and $1550 \mathrm{~nm}$.

From the graphs, sensitivity of the uric acid is calculated as shown in Table 2. Although the concentration of the albumin protein is factor of three higher than that of uric acid, the uric acid sensitivity is factor of two higher than that of albumin.

\section{Extracting uric acid concentration from uric acid + serum bo- vine solution}

Based on linear theory of the Beer Lambert's law, total light absorption is the linear superposition of the light absorbance by each molecule within the solution and can be expressed as

$$
A\left(\lambda_{1}\right)_{\text {total }}=k_{1}\left(\lambda_{1}\right) \cdot c_{1}+k_{2}\left(\lambda_{1}\right) \cdot c_{2}
$$

Where $k_{1}$ represents the spectral characteristics of uric acid and $k_{2}$ represents that of bovine albumin. Similar to $k_{1}\left(\lambda_{1}\right)_{\text {theoretical }}$ for the uric acid, $k_{2}\left(\lambda_{1}\right)_{\text {theoretical }}$ for the protein albumin can be estimated from Table 2 . By repeating measurements at different wavelength, $\lambda_{2}, A\left(\lambda_{2}\right), k_{1}\left(\lambda_{2}\right)$ and $k_{2}\left(\lambda_{2}\right)$ can be estimated. By using light absorbance and spectral characteristic information at two separate wavelengths, concentration of uric acid and bovine protein can be estimated as follows.

$$
\begin{aligned}
& A\left(\lambda_{1}\right)=k_{1}\left(\lambda_{1}\right) \cdot c_{1}+k_{2}\left(\lambda_{1}\right) \cdot c_{2} \\
& A\left(\lambda_{2}\right)=k_{1}\left(\lambda_{2}\right) \cdot c_{1}+k_{2}\left(\lambda_{2}\right) \cdot c_{2} \\
& c_{1}=\frac{A\left(\lambda_{1}\right)-k_{2}\left(\lambda_{1}\right) \cdot c_{2}}{k_{1}\left(\lambda_{1}\right)},
\end{aligned}
$$

substituting $\mathrm{c}_{1}$ to the second equation

$$
A\left(\lambda_{2}\right)=k_{1}\left(\lambda_{2}\right) \cdot\left(\frac{A\left(\lambda_{1}\right)-k_{2}\left(\lambda_{1}\right) \cdot c_{2}}{k_{1}\left(\lambda_{1}\right)}\right)+k_{2}\left(\lambda_{2}\right) \cdot c_{2}
$$

Both $\mathrm{c} 1$ and $\mathrm{c} 2$ can be estimated by this linear regression method. Calculated concentrations, $\mathrm{cl}$ and $\mathrm{c} 2$, based on absorbance measurements are compared to the actual concentration used. Protein albumin concentration of $3500 \mathrm{mg}$ was used and uric acid concentration



Figure 11: IR Sensitivity vs Wavelength.


Figure 12: Uric Acid Sensitivity.
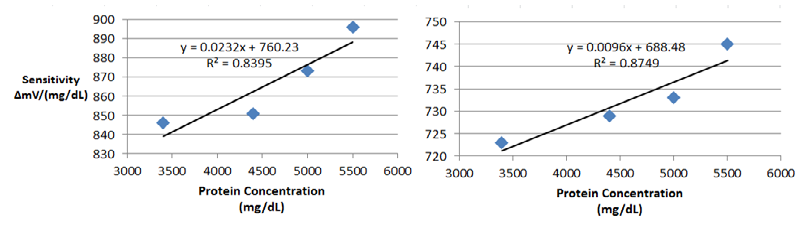

Figure 13: Albumin Sensitivity. 
Citation: Kim J (2015) Noninvasive Uric acid Monitoring Device using Near-Infrared Spectroscopy. J Biosens Bioelectron 6: 188. doi:10.4172/21556210.1000188

Page 5 of 6

\begin{tabular}{|c|c|c|}
\hline Sensitivity & $\mathbf{1 4 5 0} \mathbf{~ n m}$ & $\mathbf{1 5 5 0} \mathbf{n m}$ \\
\hline Uric Acid $(\boldsymbol{\Delta} \mathbf{V} /$ conc $)$ & 9.3 & 3.9 \\
\hline Albumin Protein $(\boldsymbol{\Delta} \mathbf{V} /$ conc $)$ & 0.023 & 0.0096 \\
\hline
\end{tabular}

Table 2: Sensitivity comparison between Uric Acid and Albumin at wavelengths 1450 and $1550 \mathrm{~nm}$.

\begin{tabular}{|c|c|c|c|c|c|c|}
\hline Uric acid + protein & $\begin{array}{c}\text { 1450nm measured } \\
(\Delta \mathrm{mV})\end{array}$ & Calculated & Error (\%) & $\begin{array}{c}1550 \mathrm{~nm} \text { measured } \\
(\Delta \mathrm{mV})\end{array}$ & Calculated & Error (\%) \\
\hline $6 \mathrm{mg}+3500 \mathrm{mg}$ & 914 & 943 & 3.075292 & 903 & 887.55 & 1.740747 \\
\hline $8 \mathrm{mg}+3500 \mathrm{mg}$ & 919 & 944.5 & 2.699841 & 910 & 891.05 & 2.126704 \\
\hline $10 \mathrm{mg}+3500 \mathrm{mg}$ & 923 & 952.5 & 3.097113 & 915 & 897.55 & 1.944181 \\
\hline $12 \mathrm{mg}+3500 \mathrm{mg}$ & 929 & 956 & 2.824268 & 918 & 901.05 & 1.881139 \\
\hline $14 \mathrm{mg}+3500 \mathrm{mg}$ & 937 & 958 & 2.192067 & 926 & 906.55 & 2.145497 \\
\hline
\end{tabular}

Table 3: Comparison between measured and calculated value.

\begin{tabular}{|c|c|c|}
\hline Uric Acid Conc. (mg) & $\begin{array}{c}\text { Average Measured Value measured } \\
\text { in (mV) }\end{array}$ & $\begin{array}{c}\text { Average Measured Value converted } \\
\text { to (mg) }\end{array}$ \\
\cline { 2 - 3 } & $(\mathbf{1 0}$ runs) & $(\mathbf{1 0}$ runs) \\
\hline $6 \mathrm{mg}$ & $1443 \mathrm{mV}$ & $5.979 \mathrm{mg}$ \\
\hline $8 \mathrm{mg}$ & $1461 \mathrm{mV}$ & $8.45 \mathrm{mg}$ \\
\hline $10 \mathrm{mg}$ & $1471 \mathrm{mV}$ & $9.91 \mathrm{mg}$ \\
\hline $12 \mathrm{mg}$ & $1477 \mathrm{mV}$ & $10.83 \mathrm{mg}$ \\
\hline $14 \mathrm{mg}$ & $1505 \mathrm{mV}$ & $17.78 \mathrm{mg}$ \\
\hline
\end{tabular}

Table 4: Repeatability analysis.

was varied from $6 \mathrm{mg}$ to $14 \mathrm{mg}$ (Table 3). IR Measurements repeatability and Error Analysis Based on measured values, spectral characteristic value ' $k$ ' was calculated.

$$
L_{\text {absorbed }}=A\left(\lambda_{1}\right)=k\left(\lambda_{1}\right) \cdot c \cdot d .
$$

Once the $k$ value is found, expected light absorption can be calculated based on the concentration used or expected concentration can be calculated based on the light absorption. For each concentration of uric acid, ten measurements were made to analyze the repeatability (Table 4).

\section{Error analysis}

Since each error source is non-correlated to each other, total error can be expressed as

$$
\begin{gathered}
\text { Total Error }=\sqrt{(\text { Linearity Error })^{2}+(\text { Repeatability Error })^{2}+(\text { Interference Error })^{2}} \\
091 \mathrm{mg}=\sqrt{(0.41 \mathrm{mg})^{2}+(0.78 \mathrm{mg})^{2}+(0.24 \mathrm{mg})^{2}}
\end{gathered}
$$

\section{Uric acid precipitation}

Through the study, it became clear why the normal recommended range of the uric acid in the blood is from 3 to $7 \mathrm{mg} / \mathrm{dL}$. As shown by Figure 14, once the uric acid concentration reaches $7.5 \mathrm{mg} / \mathrm{dL}(15 / 2)$, change in voltage measured no longer shows linear relationship but rather starts to saturate. This indicates the solution reached the maximum solubility and then precipitates start forming [12]. Once the uric acid precipitates, there will be higher chance of forming the crystals and this can be another explanation why patients with high uric acid level in the blood tend to form gouty joints more [13].

\section{Discussion}

Main objective of this project was to build a low cost Infraredbased sensor to detect the uric acid level in the solution and review its potential development as a preventative tool to measure uric acid level in the blood noninvasively. In order to accomplish the goal, prototype sensor was developed and tested. Aqueous solution was used to model the uric acid concentration in the blood, and albumin was incorporated

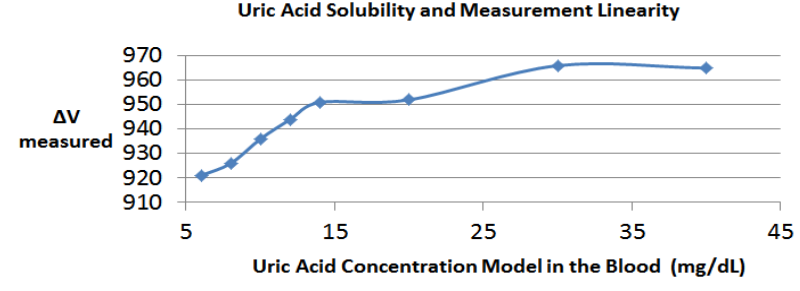

Figure 14: Uric Acid Solubility.

as realistic interfering biomarker in the blood. Future direction for this project is to incorporate actual blood samples that will allow us to deduce the uric acid concentration.

Hypothesis was based on Beer Lambert theory that the device should be able to deduce the concentration of uric acid based on the light being absorbed by the sample. Measurements were in agreement with the theory and showed the linear relationship between uric acid concentration and amount of light being absorbed. The normal uric acid concentration ranged from 3-7 mg/dL and the prototype was able to measure within the normal range with an error of $0.91 \mathrm{mg} / \mathrm{dL}$. Also, by using the linear superposition theory, [14] uric acid concentration was successfully deduced from the mixture with the protein albumin whose concentration was a factor of three higher than that of uric acid. Based on measurement data, it is indicated that the same technique can potentially be applied to the actual blood and uric acid concentration should be extractable noninvasively.

As a next step, it would be important to start adding more interfering substances such as transcutaneous model [15] and more blood interference molecules. Transcutaneous modeling will impose a great challenge as optical properties change vastly depending on the skin color, thickness, and age, [16] Adding more bio interferences will likely introduce higher non-linearity, and advanced calibration techniques will need to be incorporated accordingly to accurately 
Citation: Kim J (2015) Noninvasive Uric acid Monitoring Device using Near-Infrared Spectroscopy. J Biosens Bioelectron 6: 188. doi:10.4172/21556210.1000188

deduce concentration of the marker of interest. To tackle these limitations, skin properties with respect to the optical properties [17] will be studied extensively and a proper model will be developed on top of the current capillary tubes model.

\section{Conclusion}

An IR-based non-invasive sensor to monitor uric acid levels was developed. Portability, cost, accuracy $(0.91 \mathrm{mg} / \mathrm{dL})$, selectivity and the non-invasive nature of the sensor make the design affordable and convenient to prevent the complications associated with hyperuricemia. Accuracy of the sensor is limited by the accuracy of the infrared emitter, receiver, and data processing chips. The design is very versatile. By changing the wavelength, the sensor could potentially monitor many other bio-markers of interest.

\section{References}

1. Keenan RT, Pillinger MH (2009) Hyperuricemia, gout, and cardiovascular disease--an important "muddle". Bull NYU Hosp Jt Dis 67: 285-290.

2. Schumacher HR Jr (2008) The pathogenesis of gout. Cleve Clin J Med 75 Suppl 5: S2-4.

3. Kim SY, Guevara JP, Kim KM, Choi HK, Heitjan DF, et al. (2010) Hyperuricemia and coronary heart disease: a systematic review and meta-analysis. Arthritis Care Res (Hoboken) 62: 170-180.

4. Becker MA, Jolly M (2006) Hyperuricemia and associated diseases. Rheum Dis Clin North Am 32: 275-293, v-vi.

5. Zhu Y, Pandya BJ, Choi HK (2011) Prevalence of gout and hyperuricemia in the US general population: the National Health and Nutrition Examination Survey 2007-2008. Arthritis Rheum 63: 3136-3141.

6. Smyth CJ (1975) Disorders associated with hyperuricemia. Arthritis Rheum 18: 713-719.
7. Dugdale DC (2011) Crystal deposition diseases. Goldman L, Schafer Al, eds Cecil Medicine $\left(24^{\text {th }}\right.$ edn) Philadelphia, Pa: Saunders Elsevier chap 281.

8. Bruce M, Rothschild M (2014) Gout and Pseudogout Workup. Medscape.

9. Dalbeth N, Choi HK (2013) Dual-energy computed tomography for gout diagnosis and management. Curr Rheumatol Rep 15: 301.

10. Mendelson Y1 (1992) Pulse oximetry: theory and applications for noninvasive monitoring. Clin Chem 38: 1601-1607.

11. Yamakoshi $Y$, Ogawa M, Yamakoshi T (2007) A new non-invasive method for measuring blood glucose using instantaneous differential near infrared spectrophotometry. Conference proceedings: Annual International Conference of the IEEE Engineering in Medicine and Biology Society. IEEE Engineering in Medicine and Biology Society. Conference: 2964-2967.

12. Iwata H, Nishio S, Yokoyama M, Matsumoto A, Takeuchi M (1989) Solubility of uric acid and supersaturation of monosodium urate: why is uric acid so highly soluble in urine? J Urol 142: 1095-1098.

13. Sarawate CA, Patel PA, Schumacher HR, Yang W, Brewer KK, et al. (2006) Serum urate levels and gout flares: analysis from managed care data. Journal of clinical Rheumatology: practical reports on rheumatic \& musculoskeletal diseases 12: 61-65.

14. Swinehart DF (1962) The Beer-Lambert Law. Journal of Chemical Education 39: 333

15. Heise HM, Lampen $P$ (2000) Transcutaneous glucose measurements using near-infrared spectroscopy: validation of statistical calibration models. Diabetes care 23: 1208-1210.

16. Jacques SL (2013) Optical properties of biological tissues: a review. Phys Med Biol 58: R37-61.

17. Simpson CR, Kohl M, Essenpreis M, Cope M (1998) Near-infrared optical properties of ex vivo human skin and subcutaneous tissues measured using the Monte Carlo inversion technique. Physics in medicine and biology 43 : 2465-2478. 